\title{
Short-term wind power forecasting using the LMS algorithm
}

\author{
Abdellatif ELMANSOURI ${ }^{\# 1}$, Jamal MHAMDI ${ }^{\# 2}$ \\ ${ }^{1}$ Electrical Engineering Laboratory, ENSET, Mohamed V University, Rabat, Morocco \\ ${ }^{2}$ Pluridisciplinary Laboratory of Research \& Innovation (LPRI), EMSI Casablanca, Morocco \\ ${ }^{1}$ abdellatif.elmansouri@um5s.net.ma \\ ${ }^{1}$ mhamdi_jamal@yahoo.fr
}

\begin{abstract}
This paper presents the application of the LMS adaptive filter for the short-term wind power prediction, generated by a wind energy conversion system based on historical wind speed and wind power data, for a period of 24 hours with a step of one second. To compare the predicted power with that measured for a specific period. There are two steps in the process of predicting of wind power. In the first step, the raw data collected by the laboratory computer system. This step prepares the validated data to be used to construct an estimated model. In the second step, the LMS adaptive filter is applied to construct a model to predict wind power.
\end{abstract}

Keywords: LMS algorithm, prediction, adaptive, wind power, turbine, renewable energy.

\section{INTRODUCTION}

The world's primary reserves of polluting primary energy (oil, natural gas, coal, uranium) sooner or later it will be exhausted, while the very industrialization of the last decades its demand for electricity is always increasing. This has pushed countries towards the use of nuclear power plants to meet its energy needs. This energy source has the distinct advantage of not causing air pollution unlike thermal power plants, but the risk of nuclear accidents, waste treatment and burial are real problems that make this energy unattractive to the future generations.

Faced with this problem several countries have turned to renewable energies. Indeed, a real global challenge is taken seriously today, as well on the policy of reducing greenhouse gas emissions. These sources of renewable energy include wind energy and the one with the highest energy potential.

It is clear that Morocco is seriously engaged in this challenge with the installation of several photovoltaic power stations and wind farms whose objective is to achieve a $50 \%$ renewable energy production of national production by 2030 , Which also motivated us to direct our research work on renewable energies, specifically wind energy.

The main problem of wind power is that the primary energy (the wind) is not controllable. This means that the power output of the wind turbines will vary according to the wind speed. It is therefore necessary to implement solutions in order to allow the manager to limit the impact of these productions on electric network [1].

Therefore, the objective of this work is to implement an efficient prediction method based on adaptive filters to provide an estimate of the output power of a wind turbine for prediction horizons not exceeding the hour. This will enable the manager in the future to optimize the management of his electricity network by facilitating the integration of the wind resource [2].

\section{Problem}

One of the main factors contributing to slowing down the integration of wind energy into energy distribution networks is the variation of the power produced. Indeed, the energy production of a wind power plant depends on weather conditions that vary randomly. It is therefore impossible to control the power produced by the wind turbines [3]. Operators of the grid power which must meet the energy demand need to make forecasts of distribution of electric energy several days in advance in order to maintain the electric network balanced at all times. In this context, developing tools capable of predicting the output power of wind turbines several hours ahead is a key solution, to enable this source of energy to be more easily integrated into grid energy distribution. This solution could also help to increase its economic value, which is affected by its variation. Several mathematical methods are used in wind power prediction, such as the autoregressive moving average process, adaptive filters, artificial neural networks and methods based on meteorological forecasts digital. The latter methods use of autoregressive models based on the use of time-indexed data history called time series. The analysis of the wind speed series shows that we are dealing with a stochastic process. Indeed, these wind measurements are presented as a sequence of random variables indexed by time [4]. 


\section{SUGGESTED SOLUTION}

The adaptive methods in signal processing aim at the automatic adaptation of the processing operators to the statistical properties of the signals and the systems, as well as the adaptation to their variations over time. It is therefore a well-balanced mixture between stationarity. Which to get rid of, or at least to reduce statistical properties, or the purely random fluctuations, and non-stationarity, meaning the "slow" variation over time of these properties [5].

A filtering is made adaptive if its parameters and the coefficients are modified according to a given criterion, as soon as a new value of the signal becomes available. These changes must follow the evolution of the systems in their environment as quickly as possible. Adaptive filtering is generally associated with real-time operation, figure (1) shows principle of an adaptive filter.

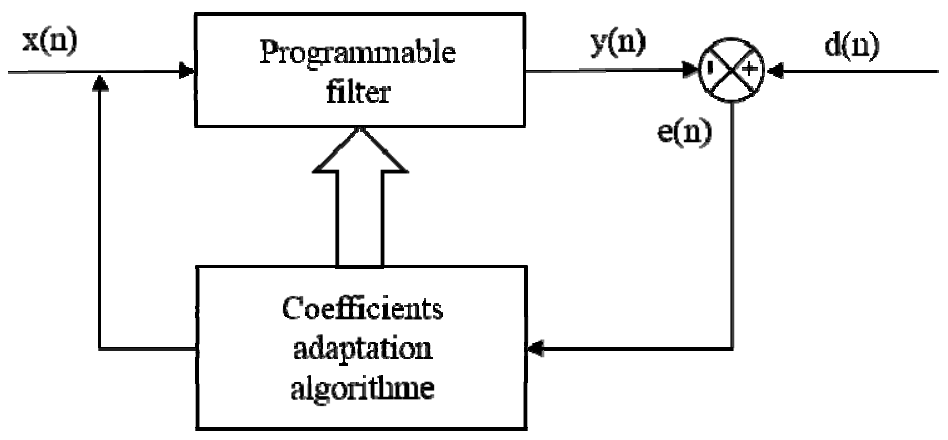

Fig.1. Principle of an adaptive filter

Adaptive filters can be classified according to the choices made on the following points [6]:

- The optimization criterion

- The algorithm for the coefficients updating

- The structure of the programmable filter

-The type of processed signal, mono or multidimensional.

There are two important classes of optimal linear filters:

- Wiener filtering (where the considered signals $\mathrm{d}(\mathrm{n})$ and $\mathrm{x}(\mathrm{n})$ are stationary),

- Kalman filtering (which is a generalization of the Wiener filter also valid in the case of non-stationary processes (or signals)).

\section{IV.CHOICE OF THE ALGORITHM}

The choice of the algorithm will be made according to the following criteria:

- The speed of convergence, which will be the number of iterations necessary to converge "close enough" to the optimal solution

- The measurement of this "proximity" between this optimal solution and the solution obtained

- The tracking capability of the system's non-stationary variations

- Robustness to the noise

-The complexity

- The structure (modularity, parallelism ...)

- The numerical properties (stability and precision) in the case of a limited precision on the data and the coefficients of the filter [7].

\section{Digital apPlications}

Adaptive filtering is a powerful tool in signal processing, digital communications, and automatic control. The applications are diverse but have the following characteristics: there is an input $\mathrm{x}(\mathrm{n})$ as well as the desired response d (n) and the error e (n) (e (n) is the difference between d (n) and the output of the filter y (n)), serves to control the values of the filter coefficients. This essentially differentiates applications that come from the way we define the desired response $d(n)$. There are four main classes of applications [8]:

- Identification of systems

- Prediction

- Reverse Modeling

- Interference cancellation 


\section{VI.THE LMS FILTERING METHODOLOGY FOR THE PREDICTION OF WIND POWER}

\section{A. The LMS algorithm}

In this work, we are interested in the application of adaptive filters in the field of prediction, so we want to predict the power delivered by a $3 \mathrm{~kW}$ wind turbine with horizontal axis from historical data recorded for 24 hours with a time step of a second.

This algorithm is very simple, which is the most popular adaptive algorithm. Its complexity is $\mathrm{M}$ multiplications by iteration [9].

Calculation of filter output

$y_{n}=h^{T}(n) x(n)$

Calculation of the error

$$
e(n)=d(n)-y(n)
$$

Filter update

$$
h(n+1)=h(n)+\mu x(n) e(n)
$$

Tap weight vector

$$
\bar{w}=\left[w_{0}, w_{1}, w_{2}, \ldots, w_{N-1}\right]^{T}
$$

\section{Signal input}

$$
\overline{x(n)}=[x(n), x(n-1), x(n-2), \ldots, x(n-N+1)]^{T}
$$
2), ....

$d(n)$ is the signal at time (n) and y (n) the signal predicted from the signal at the previous instants $d(n-1), d(n-$

The function (2) represents the error between the filter output and the reference. A simple LMS algorithm is used to minimize this error. The figure (2) shows the principle of prediction by an adaptive filter.

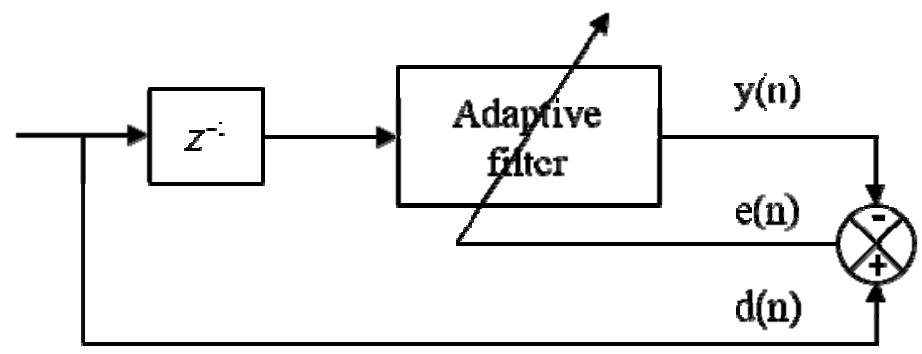

Fig.2. Principle of prediction

The optimal filtering problem is to find the "best" filter, which allows to obtain at the output y(n) response as close as possible to a desired response $d(n)$ when the input is a given sequence $u(n)$. [10].

The error between the desired response $d(n)$ and the output $y(n)$ is denoted by e $n)=y(n)-d(n)$. We also note $h$ (n) the impulse response of the filter.

A simple algorithm can be written as:

fori=1:nk,

$e(i)=d(i)-w(i) * x(i)$;

$\mathrm{w}(\mathrm{i})=\mathrm{w}(\mathrm{i})+(\mathrm{m} * \mathrm{e}(\mathrm{i}) * \mathrm{x}(\mathrm{i}))$

end.

Where $\mathrm{e}, \mathrm{x}$ and $\mathrm{w}$ are defined as previously and $\mathrm{m}$ is the adaptation rate.

B. Convergence of the LMS algorithm:

To analyze the convergence of the LMS, we introduce the filter error as follows:

$e(n)=d(n)-y(n)$

The convergence factor must satisfy the following inequality:

$0<\mu<\frac{1}{N \times \text { Maximum input power }}$ 


\section{Stability of LMS}

The LMS algorithm is convergent in the mean square if and only if the step-size parameter satisfy

$0<\mu<\frac{2}{\lambda_{\max }}$

Here $\lambda_{\max }$ is the largest eigenvalue of the correlation matrix of the input data.

Extra practical test for stability is given by:

$$
0<\mu<\frac{2}{\text { input.signal.power }}
$$

Large values for step size increase adaptation rate (faster adaptation) and increase residual mean-squared error.

\section{Choice of filter order}

The choice of an optimal order for an adaptive filter model is made according to a compromise between the complexity of the model chosen and the residual error of identification. Akaike (1973) proposes two criteria: FPE (Final Prediction Error) and AIC (Akaike Information Criterion). The FPE is defined by [11]:

$$
\operatorname{FPE}(p)=\frac{N+(p+1)}{N-(p+1)} \sigma_{p}^{2}
$$

Where the $N$ Represents the length of the signal, $p$ the filter order and $\sigma^{2}$ is an estimator of the variance of the noise.

The AIC criterion can be formulated as:

$$
A I C=\frac{2 N}{N}+\operatorname{Ln} \sigma_{p}^{2}
$$

Based on the AIC function (9) to choose the model with the lowest Akaike information criterion. The order obtained corresponds to $\mathrm{p}$ being around 20.

\section{VII.THE PLATFORM AND DATA DESCRIPTION}

\section{A. Platform description:}

The wind system is installed on the roof of Moroccan school of engineering sciences in Casablanca (latitude $=33.5415060$ and longitude $=-7.6735389)$. This platform was constructed for the research and educational purposes. The platform is made up of two wind turbines. One with horizontal axis and the other with vertical axis both produce a power of $3 \mathrm{~kW}$, connected to the electrical network of the school, figure (3) present the EMSI wind system platform.

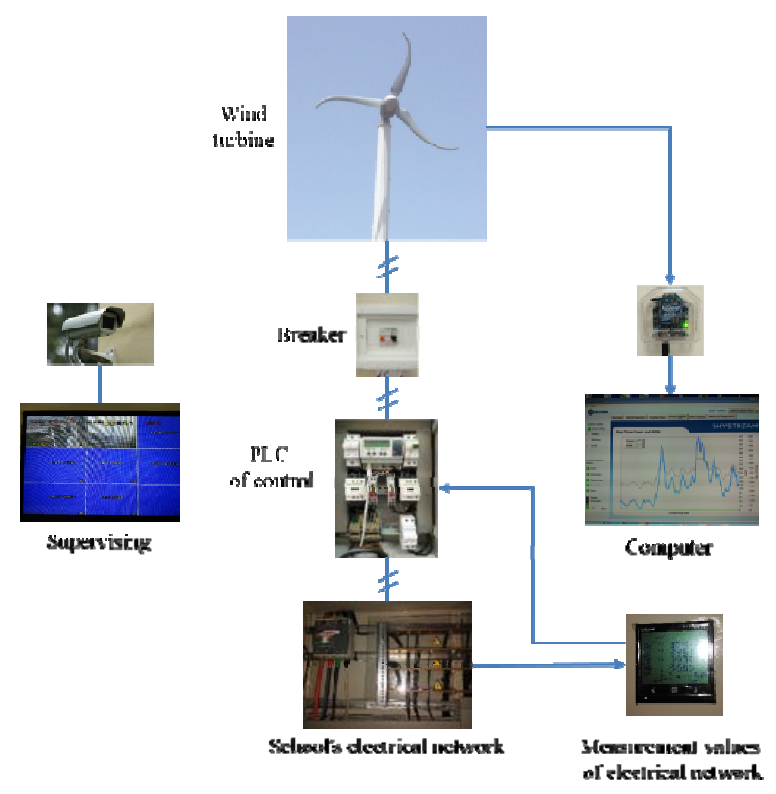

Fig.3. EMSI Wind system platform 


\section{B. Data description and analysis:}

The study is executed on a turbine of a $3 \mathrm{Kw}$; it is a three bladed horizontal axis model. The following table presents the wind turbine data. Table I present de wind turbine data.

TABLE I. The wind turbine data

\begin{tabular}{|c|c|c|}
\hline Signification & Symbol & Value \\
\hline Blade Radius & $R$ & $3 \mathrm{~m}$ \\
\hline Base wind speed & $V$ & $12 \mathrm{~m} / \mathrm{s}$ \\
\hline Power coefficient & $C_{p}$ & 0.48 \\
\hline Optimal relative wind speed & $\lambda$ & 8.1 \\
\hline Moment of inertia & $J$ & $0.3125 \mathrm{~kg} / \mathrm{m}^{2}$ \\
\hline
\end{tabular}

The data of wind speed $(\mathrm{m} / \mathrm{s})$ and output power $(\mathrm{w})$. Are monitored by sensors installed at the tower. All the acquired data is averaged over a one-second period for turbine power curve measurement. The following table describes the data history of wind speed and electrical power.

TABLE II. Data description

\begin{tabular}{|c|c|c|c|c|}
\hline Data group & Start time & End time & No. of Data points & Description \\
\hline A & $23 / 03 / 201623: 59$ & $24 / 03201612: 00$ & 6725 & Training data set \\
\hline B & $23 / 03 / 201612: 01$ & $24 / 03 / 201623: 59$ & 6724 & Testing data set \\
\hline
\end{tabular}

The figure (4) presents a plot of data wind speed and output power.

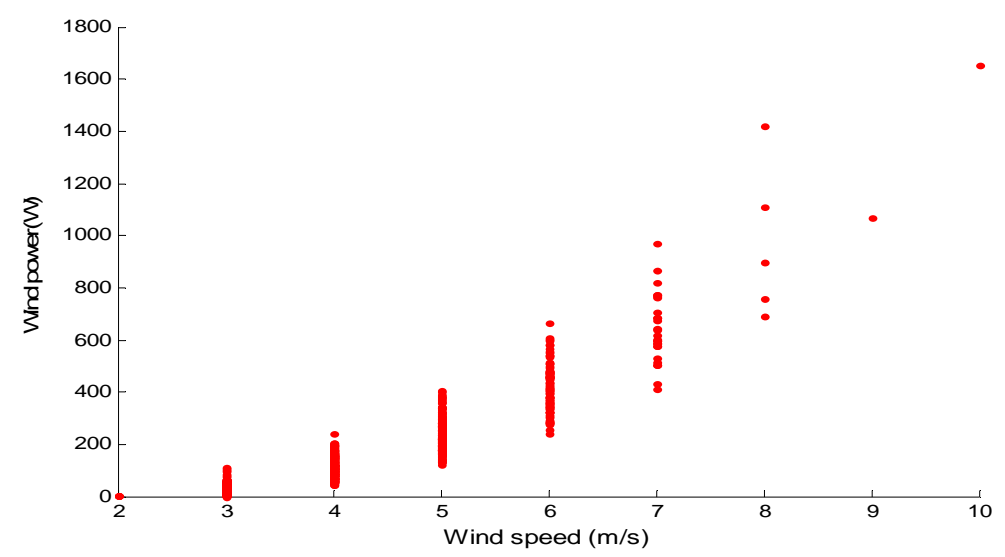

Fig.4. Group data of power and wind speed.

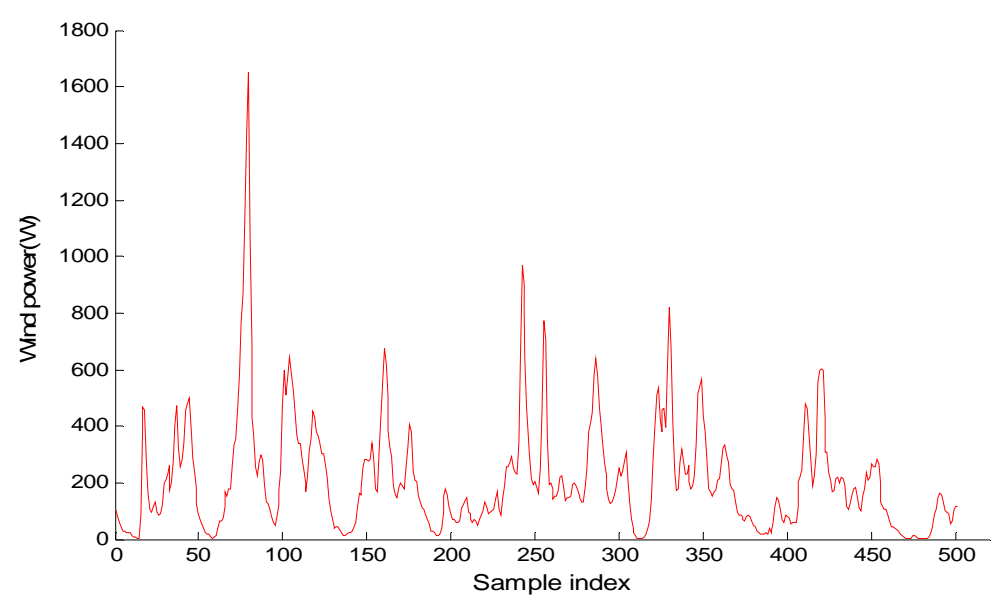

Fig.5. Plot of power data points.

Figure (5) shows the appearance of several points for which the power of the wind turbine has a negative or zero value. These data correspond to the stops of the turbine. 


\section{SiMULATION RESULTS}

The suggested solution was simulated using (Matlab / Simulink). Figure (6) shows the predicted power curve and the reference; while Figure (7) shows the same results but within a reduced range to clearly see the margin error between the reference and the measurement. The adaptation step used here is $\mu=0.00000003$.

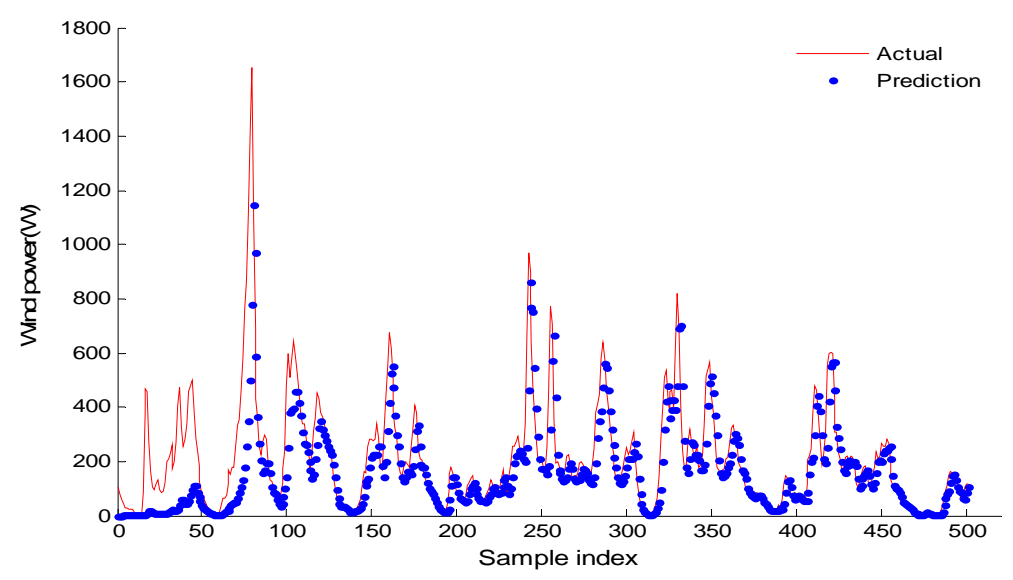

Fig.6. Wind turbine output power variation of predicted and data power with $\mu=0.00000003$.

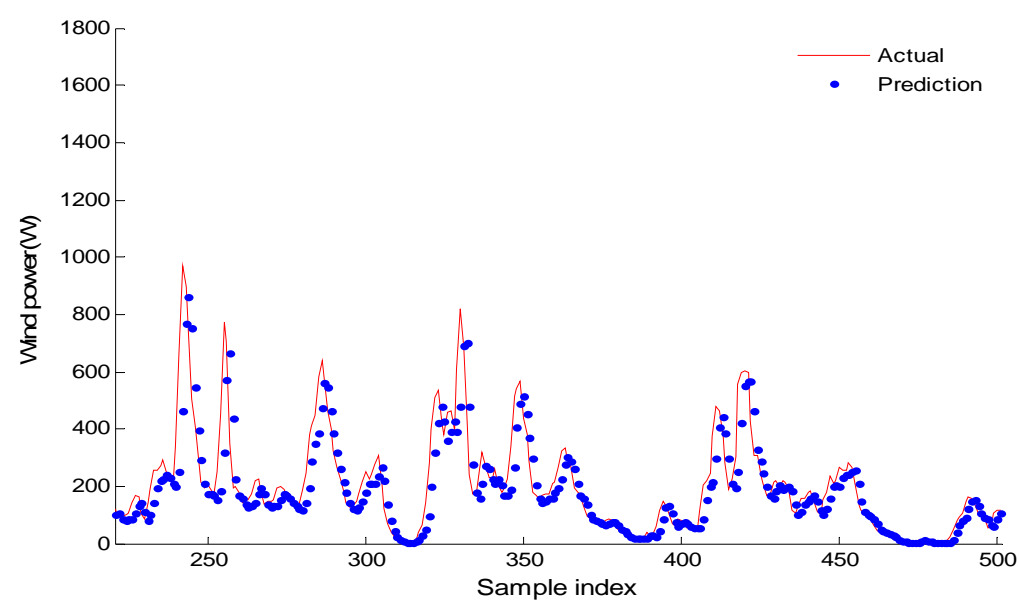

Fig.7. Zoom of wind turbine output power variation of predicted and data power with $\mu=0.00000003$.

So we can see correctly that the predicted curve follows the reference with an acceptable margin of error. The results of the following simulations also show the predicted power and the reference, but this time with a greater adaptation step than the first one $\mu=0.000003$.

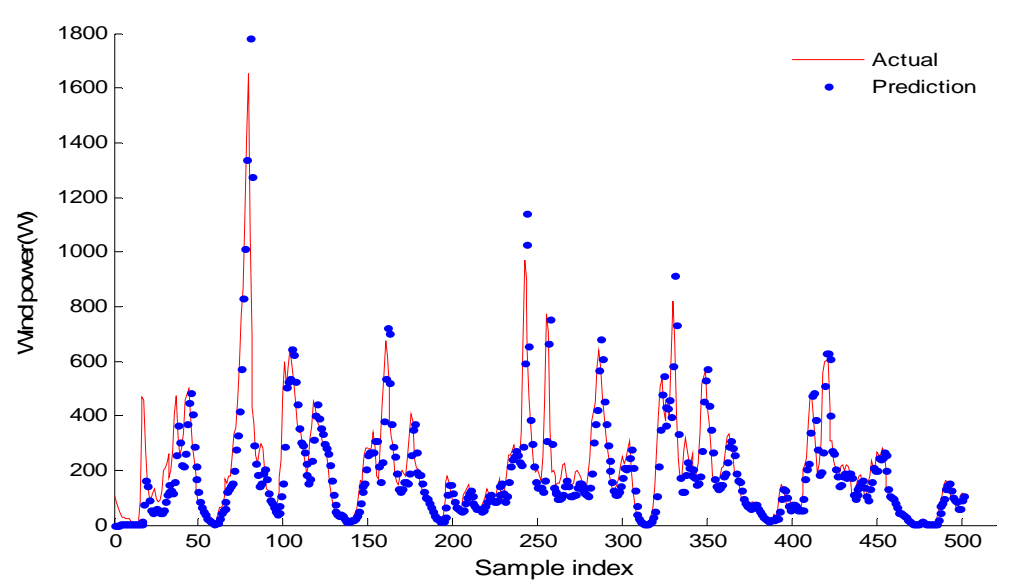

Fig.8. Wind turbine output power variation of predicted and data power with $\mu=0.00003$. 


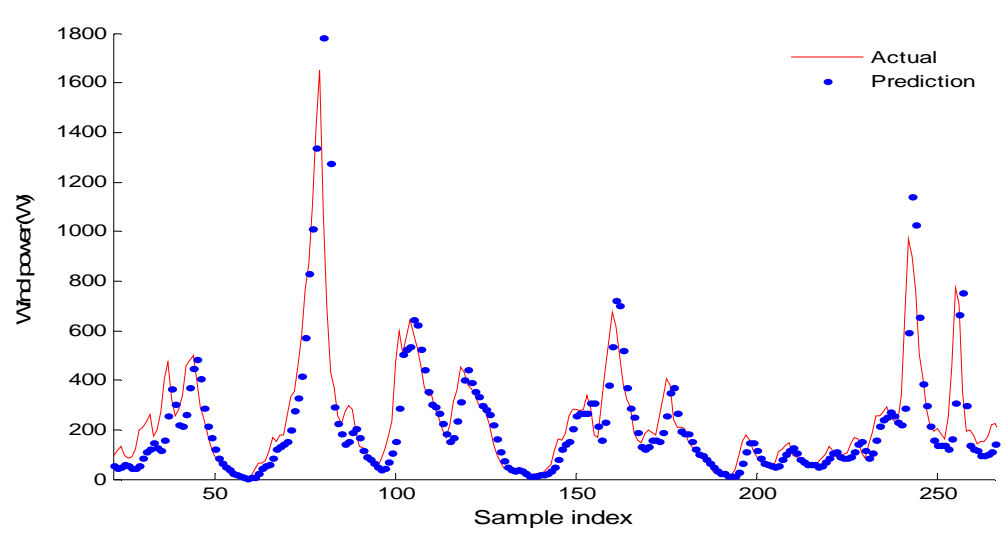

Fig.9. Zoom of wind turbine output power variation of predicted and data power with $\mu=0.00003$.

From Figures (8) and (9) it can be seen that when the adaptation step has been changed to a larger one. The algorithm converges more rapidly compared with the one that has a smaller step, but the residual error is greater.

\section{IX.CONCLUSION}

The first part of this work is devoted to the presentation of the LMS algorithm and its application to the prediction of the power curve of a wind turbine. While the second part presents the platform of renewable energies of the Moroccan School of engineering Sciences and the description of the historical data. Finally, simulation results show correctly that the predicted curve follows the reference with an acceptable margin of error and it can be seen that when the adaptation step has been changed to a larger one. The algorithm converges more rapidly compared with the one that has a smaller step, but the residual error is greater.

\section{REFERENCES}

[1] Ling Xiao, JianzhouWang, YaoDong, JieWu, Combined forecasting models for wind energy forecasting: A case study in China, in the Renewable and Sustainable Energy Reviews,pp 271 - 288, 2015.

[2] Aoife M. Foley, Paul G. Leahy, AntoninoMarvuglia, Eamon J. McKeogh, Current methods and advances in forecasting of wind power generation, in the Renewable Energy , Volume 37, Issue 1, pp. 1-8, January 2012.

[3] José Antonio, Medina Hernández; Felipe Gómez Castañeda; José Antonio Moreno CadenasNumerical, "'Study of Least Mean Square Method for Adjusting Curves", inInternational Conference on Electrical Engineering, Computing Science and Automatic Control (CCE),pp 256 - 261, 2013.

[4] Muhammad Khalid, Andrey V. Savkin. Adaptive filtering based short-term wind power prediction with multiple observation points. In International Conference on Control and Automation, pp 1547 - 1552, 2009.

[5] Mahdi Tohidian, A. Esmaili, Ramezan-Ali Naghizadeh, S.H.H. Sadeghil, A. Nasire, Ali M. Reza, Use of Adaptive Linear Algorithms for Very ShortTerm Prediction of Wind Turbine Power Output, in IECON 2012 - 38th Annual Conference on IEEE Industrial Electronics Society, pp. $1162-1165,2012$.

[6] Simon S. Haykin, Bernard Widrow (Editor): Least-Mean-Square Adaptive Filters, Wiley, 2003.

[7] E. S. Jin, L. L. Liu, Z. Q. Bo and A Klimek, Parameter Identification of the Transformer Winding based on Least-Squares Method, in IEEE Power and Energy Society General Meeting - Conversion and Delivery of Electrical Energy in the 21st Century, pp. 1-6, 2008.

[8] Yun ZHAO, Xiaoming LI, Lingxu AN, Jian SUN, Lihui WANG, Research on encoding/decoding method of electric physical information based on LMS-ADPCM algorithm, in The International Conference on Advanced Power System Automation and Protection, pp. $795-800,2011$.

[9] Rashpinder Kaur, Gurjinder Singh, Balwinder Singh Dhaliwal, Sandeep Singh Gill, Implementation of Modified Variable Step Size Least Mean Square Algorithm in Lab VIEW, in International Conference on Computational Intelligence and Computing Research, pp. $1-4,2012$.

[10] EleftheriosGiovanis, Applications of Least Mean Square (LMS) Algorithm Regression in Time-Series Analysis, in Munich Personal RePEc Archive, Paper No. 24658, August 2010.

[11] Akaike H. 1969, Power spectrum estimation through autoregressive model fitting. Ann. Inst. Statist. Math., 21, 407-419. 


\section{AUTHOR PROFILE}

Abdellatif ELMANSOURI PhD student, was born in 1987 in Morocco, he received her Master's degree in ElectricalEngineering from Higher Normal School of Technical Education (EcoleNormaleSupérieure de l'EnseignementTechnique - ENSET), Rabat Mohammed V University, Morocco, in 2012. he is actually a PhD student since2013 in Sciences and Technology of the Engineer in the National School of Computer Science and SystemsAnalysis (EcoleNationaleSupérieured'Informatique et d'Analyse des Systèmes - ENSIAS), ResearchLaboratory in Electrical Engineering LRGE, located in the Higher Normal School of Technical Education ENSET, Mohammed V University, Rabat, Morocco. He is author or co-author of several papers in international conferences and journals. His researchinterests include the renewable energy.

Jamal ELMHAMDI received the master degree EEA in 1984 and the PhDin signal processing and Telecommunications by the University of Rennes (France) in 1988 he was professor in the Mohammed V University in Morocco. He manages theTeam of Research in Computer Science and Telecommunications. He is currentlyprofessor inHigher Normal School of Technical Education (EcoleNormaleSupérieure de l'EnseignementTechnique - ENSET), Rabat Mohammed V University, Morocco. He is author or co-author of several papers in international conferences and journals.His domains of interest include signal processing and telecommunications. He is also a member of the reading committee of several international conferences and journals. He has supervised and co-supervised several doctoral theses. His research interests include the problems of implementing signal processing algorithms and image on DSP and FPGA in the real-time embedded systems. 